\title{
AlexandRa Aidler
}

Peter L. Oesterreich: Spielarten der Selbsterfindung. Die Kunst des romantischen Philosophierens bei Fichte, F. Schlegel und Schelling, Berlin/New York: De Gruyter 20 I I (= Untersuchungen zur deutschen Literaturgeschichte I 39). 235 S. € 79,95. ISBN 978-3-I I-025 I 23 -4

Die Philosophie Johann Gottlieb Fichtes lobt Friedrich Schlegel in einem im Jahre 1799 veröffentlichten Aufsatz für ihre durch $\gg$ Popularität und Klarheit $\ll^{1} \gg$ ins Unendliche fortgesetzte[] Reflexion $\ll .^{2}$ Laut Schlegel wird in der Wissenschaftslehre Fichtes die individuelle Neigung zu einem »künstliche $[n]$ Denken $\ll^{3}$ mit dem Geist der » allgemeinste $[\mathrm{n}]$ Mitteilung « zusammengeführt. ${ }^{4}$ Zwischen dem Willen zur Selbstgestaltung und der universellen Mitteilbarkeit oszillierend, deutet Schlegel den Idealismus Fichtes als Beispiel eines Denkens, in dem das Streben nach persönlicher Transformation über den philosophischen Szientismus bis hin zur »Humanität « ${ }^{5}$ führt. Im Gedankengebäude Fichtes, so Schlegel, entspringt das gemeinschaftliche Bewusstsein aus der Entfaltung des Subjekts innerhalb der Grenzen der Philosophie.

In seinem Buch Spielarten der Selbsterfindung begibt sich Peter L. Oesterreich auf das Terrain einer romantischen Interpretation des deutschen Idealismus. Dem von Georg Wilhelm Friedrich Hegel über die Romantik gefällten Urteil der » >Absolute[n] Sophisterei< « (S. 47) widersprechend, sucht diese Studie die vom Idealismus hergeleitete »Romantikrepugnanz « (S. 2) durch den Nachweis der Verbindung von Romantik und Idealismus zu überwinden. Oesterreich knüpft damit implizit an die über zwei Jahrhunderte zuvor angestellten Beobachtungen Friedrich Schlegels zur philosophischen Wissenschaftlichkeit, dem persönlichen Erfindungsdrang und der zwischenmenschlichen Kommunikation im Idealismus an. Aus einer romantischen Perspektive will Spielarten der Selbsterfindung auf den gemeinsamen Nährboden zweier philosophischer und literaturwissenschaftlicher Strömungen hinweisen, die trotz ihrer gemeinsamen historischen Herkunft »disziplinär $\ll(S .3)$

1 Friedrich Schlegel: Über die Philosophie. An Dorothea. In: KFSA 8, S. 41-62, hier: S. 57 .

2 Ebd.

3 Ebd., S. 7. Hervorhebung i. d. Vorl.

4 Ebd., S. 57. Hervorhebung i. d. Vorl.

5 Ebd., S. 58. Hervorhebung i. d. Vorl.

Peter L. Oesterreich: Spielarten der Selbsterfindung 
222 bislang überwiegend als antithetisch aufgefasst wurden. Oesterreich plädiert für die Überwindung eines längst überholten IdealismusBegriffes (S. IX ff.). Anstatt den Idealismus Immanuel Kants, Fichtes oder Friedrich Wilhelm Joseph Schellings als einen »philosophischen Sonderweg « (S. I ) zu definieren, der sich in seiner »ernstgetragenen Systemphilosophie « (S. I) von der mit der spielerischen Ironie verwandten Romantik eines Friedrich Schlegel abgrenzt, weist der Autor auf die romantische Konvenienz mit dem Idealismus hin.

Ausgehend von einer Verflechtung von Fachphilosophie, Literaturwissenschaft und Rhetorik (S. IX) wie auch von einer Engführung einer bestimmten Form der philosophischen Anthropologie mit der » Figur der Ironie « (S. IX) skizziert Spielarten der Selbsterfindung ein Porträt der » >Kunst des romantischen Philosophierens $<\ll$. Ihr Hauptanliegen sieht Oesterreich in dem Entwurf von »transgressiven Autoinvenienz-Projekte $[\mathrm{n}] \ll($ S. I 4). Die Romantik macht es sich demzufolge explizit zum Ziel, das von der Renaissance bis hin zur Philosophie René Descartes' befürwortete anthropologische Konzept der »Selbsterfindung « (S. 4) durch seine Steigerung nach Innen zu radikalisieren. Bis zur Romantik, so lautet die These, war die » moderne[] Subjektivität « (S. 5) primär an der Erfindung der »äußeren Kulturwelt « (S. 5) interessiert. Erst das romantische Menschenbild legt seinen Schwerpunkt auf die literarische Konstruktion des »eigenen, inneren Selbst « (S. 5).

Nachgezeichnet wird in Spielarten der Selbsterfindung der von der Romantik anvisierte Transfer des anthropologischen Programms der subjektiven Selbstgestaltung in den Bereich der Ästhetik anhand von drei exemplarischen Studien zu Fichte, Friedrich Schlegel und Schelling. Über die unterschiedlichen Profile der drei Autoren zeigt Oesterreich, wie das permanente Wechselspiel von »>Selbstschöpfung und Selbstvernichtung $\ll$ (S. 7) seinen Niederschlag in einer »Dynamik « (S. 9) der rhetorischen Pole der $\gg$ infinite[n] Ironie und synekdochische[n] Ernstsetzung « (S. 9) findet. Der Autor skizziert ein romantisches Subjekt, das sich zwischen der ironischen Selbstzersplitterung und dem synekdochischen Wunsch, sich als Teil einer »organologischen Totalität « (S. 8) zu verstehen, bewegt. Vor einer Ironie, die sich in »phantastische[r] Selbstverirrung « (S. 5) zu verstricken droht, flüchtet deshalb auch das romantische Denken in ein ernsthaftes Ganzes, etwa die $>$ Nation $<$, die $>$ Geschichte $<$, das $>$ Christentum $<$, oder auch den $>$ Tod $<$ und das $>$ Böse $<$ (S. I 2).

Mit seinem Buch Spielarten der Selbsterfindung blickt Oesterreich zugleich auf die Verflüchtigung der >infiniten Ironie < in der ersten Hälfte 
des I 8. Jahrhunderts. ${ }^{6}$ In einer tour d'horizon zwischen dem Früh- und 223 Spätwerk Fichtes, Schlegels und Schellings zeigt der Autor den Triumph der ironischen Umkehrung. Bei Fichte manifestiert sich die kreative Moderation in der Hinwendung zur starken deutschen Nation (S. 45), Schlegel rekurriert auf eine » >liebevolle[] Ironie < « (S. 79), die im Projekt eines christlichen Spiritualismus des Wortes (S. 73 ff.) gipfelt, während Schelling an einer Philosophie des menschlich Bösen (S. I I 6 ff.) den dualen Charakter der menschlichen Selbstgestaltung dramatisiert. Von einer ironischen Anthropologie der Freiheit, von einer kritischen Distanznahme gegenüber den bürgerlichen Konventionen oder von einer subjektiven Befürwortung des »revolutionären Zeitgeist $[$ es $] \ll(S$. I 5$)$ verabschiedet sich die Philosophiegeschichte laut Oesterreich mit der » provokativen literarischen Inszenierung [der] pessimistischen Willensmetaphysik « (S. 207) Arthur Schopenhauers.

Mit dem Umschlag von einem »emanzipatorischen Prozess « (S. 65) der Selbsterfindung zu dezidiert konservativeren Modellen der gemeinschaftlichen Zugehörigkeit erzählt Spielarten der Selbsterfindung über einen nahezu unsichtbaren Faden die politische Geschichte einer sich in der ersten Hälfte des I 9. Jahrhunderts vollziehenden » rhetorischen Wendung (rhetorical turn $)_{\ll}($ S. 2). Für Oesterreich beginnt die soziale Performanz der romantischen $\gg$ Tropologie der Anthropologie « (S. I26) mit der Prägung eines neuen literarischen Raumes, in dem der Fokus explizit auf das »kommunikative Gelingen « (S. I 38 ) der Selbstgestaltung gelegt wird. Sowohl die infinite Ironie als auch die ernsthafte Synekdoche müssen durch literarische Innovation kollektiv auf Gehör stoßen. Die Romantik will nach Oesterreich durch offene literarische Formen jeden Menschen zur Gestaltung seiner selbst erziehen. Nicht allein dem Philosophen soll die Konstruktion des Ich vorbehalten sein, sondern durch eine Vielfalt an literarischen Gattungen soll eine publizitäre Sphäre etabliert werden, die das Subjekt zur Selbsterfindung verleitet. Insbesondere in den von Schelling eingesetzten Formen »Traktat, Vorlesung, Briefform, Dialog und Mythos « (S. I 08$)$, im »Fragment « Friedrich Schlegels (S. 6I) und im »narrative [n] Modus « der $>\gg$ Erzählung $<\ll($ S. 20) von Fichtes Wissenschaftslehre und Reden an die deutsche Nation kristallisiert sich laut Oesterreich die romantische Öffentlichkeit.

6 Zum Vergleich auch: Matthias Schöning: Ironieverzicht. Friedrich Schlegels theoretische Konzepte zwischen Athenäum und Philosophie des Lebens. Paderborn u. a. 2002.

Peter L. Oesterreich: Spielarten der Selbsterfindung 
224 Die Studie Oesterreichs überzeugt durch ihren methodologischen Ansatzpunkt. Wiederholte Motivaufnahmen und nuancierte Verschiebungen der vielschichtigen Verschränkung von Literatur, Philosophie, Rhetorik und Anthropologie lassen die Subjektphilosophie Fichtes, Schellings und Schlegels als ein einheitliches Gebilde mit einem ähnlichen Interesse an der ironischen Konstruktion des Subjekts erscheinen. Die $»$ romantische Transformationsrhetorik « (S. I 69) stellt in Spielarten der Selbsterfindung selbst einen $>$ Sonderweg $<$ innerhalb der Entwicklung der Literaturwissenschaft und der Philosophie dar. In ihrem Insistieren auf der literarischen Entfaltung der » menschlichen Freiheit « (S. I I 6) setzt sie sich über die seit der griechischen Tradition befürworteten rhetorischen »Idee der philosophischen Bildung « (S. 17 I ) hinweg. Die Romantik, so argumentiert Oesterreich, bricht mit der persuasiven Performanz. Sie nimmt ihren Inhalt nicht aus »einer bloßen Belehrung (docere) « (S. I 69), die auf eine $\gg$ die gesamte Persönlichkeit revolutionierende Bewegung (movere) (S. 169) in allen Bereichen abhebt, sondern sie versucht das Subjekt über die » metaphysisch legitimierte[] Freisetzung der künstlerisch kreative[n] Selbstbestärkung « (S. I 42) in Szene zu setzen. In der Romantik wird mit der abendländischen Tradition der sophistischen Manipulation der Rede einerseits und mit der platonisch-sokratischen $\gg$ psychagogischen Universalrhetorik « (S. 172) andererseits gebrochen.7 Spielarten der Selbsterfindung zeigt, wie die Romantik Fichtes, Schlegels und Schellings das Subjekt selbst zum Zentrum der Beständigkeit werden lässt, ohne dabei der Normativität zu verfallen.

Lediglich der Raum für eine systembezogene Lektüre bleibt in Spielarten der Selbsterfindung offen. Im Hinblick auf das philosophische Verhältnis zwischen den drei Autoren kann die Frage nach der gedanklichen Nähe von Romantik und Idealismus wieder aufgeworfen werfen. So könnte zum Beispiel eine stärker philologisch ausgerichtete Deutung hervorheben, dass Fichte und Schelling bereits im Jahre 1799 insbesondere vor dem Hintergrund ihrer gravierenden philosophischen Differenzen den Kontakt abbrechen: Während Fichte das Absolute in einer Verknotung zwischen Philosophie und Ich sucht, betrachtet Schelling das Absolute als das Derivat einer Interferenz zwischen Natur und Ich. ${ }^{8}$ Ebenfalls kann an die Polemik Schlegels gegen Fichtes Philosophie des absoluten Ich ab

7 Dazu auch François Châtelet: Une histoire de la raison. Entretiens avec Émile Noël. Paris 1992, S. 42.

8 Johann Gottlieb Fichte, Friedrich Wilhelm Joseph Schelling: Schelling-Fichte Briefwechsel. Hg. v. Hartmut Traub. Neuried 200 I, S. 179; S. I9I. 
dem Jahre i 808 erinnert werden. ${ }^{9}$ Nicht zuletzt könnte auch der Einwand 225 erhoben werden, dass gerade die von Oesterreich identifizierte nationale, geschichtliche und religiöse Wende der hier erwähnten Autoren unterschiedlich ausfällt. Setzt sich Fichte für ein christliches Deutschland ein, so versucht Friedrich Schlegel etwa seine politische Philosophie mit einer metaphysischen Monarchie in Einklang zu bringen, während Schelling auf eine politikskeptische Haltung rekurriert, die sich vom realen Staat abwendet. ${ }^{10}$

Anregend bleibt Spielarten der Selbsterfindung trotz dieses Desiderats. Die von Peter L. Oesterreich vorgezeichnete Dichotomie zwischen Romantik und Systemphilosophie wirft auf durchaus subtile Weise der Ideengeschichte einen Hegelianismus vor, der das besondere Verdienst der Romantik nicht würdigen konnte. Aufschlussreich ist die Studie auch aufgrund ihrer Darstellung der rhetorischen Innovation der Romantik um I 800 . Auf die Verstrickungen des klassischen »Res-verbum-Integralismus « (S. 209) und den sophistischen Kunstgriff andererseits bietet die ironische Subjektivität der Romantik eine kommensurable Antwort. Spielarten der Selbsterfindung eröffnet damit neue Perspektiven im Intervall zwischen Philosophie und Literaturwissenschaft.

9 Friedrich Schlegel: Fichte. 1808. In: KFSA 8, S. 63-85.

$10 \mathrm{Zu}$ den unterschiedlichen Staatsauffassungen Fichtes und Schellings auch: Günter

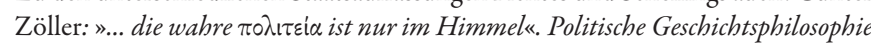
im Spätwerk Fichtes und Schellings, Brüsseler Kongress der Internationalen FichteGesellschaft (2009). Plenarvortrag. www.europhilosophie.eu/recherche/IMG/ pdf/Plenar-Zoller-beitrag.pdf. Zuletzt aufgerufen am 04.06.20 I 2. Hier besonders Seite: $16-17$.

Peter L. Oesterreich: Spielarten der Selbsterfindung 
